\title{
"Mirarse al espejo": Construcción de la identidad profesional del maestro en las prácticas escolares
}

Datos de contacto:

Miquel Alsina Tarrés Universidad de Girona, España. miquel.alsina@udg.edu

Alexander Masardo University of Gloucestershire, UK. amasardo@glos.ac.uk

Recibido: $17 / 04 / 2021$ Aceptado: 06/09/2021

\author{
Miquel ALSINA TARRÉS \\ Alexander MASARDO
}

\section{RESUMEN}

La construcción de la identidad profesional ocupa un lugar complejo entre los componentes de la formación docente. A su vez, las prácticas escolares, en la formación inicial del maestro, suponen un contexto fundamental para el desarrollo identitario del estudiante, con singularidades y diferencias notables según el contexto institucional donde se desarrollan. En este artículo se comparan las prácticas externas en los grados de magisterio en España e Inglaterra, tomando como ejemplo los casos de las universidades de Girona y Gloucestershire. A continuación, se ofrecen los resultados de un trabajo sobre la construcción de la identidad docente, en un grupo de maestros y maestras en formación de segundo curso $(\mathrm{N}=16)$ participantes en un proyecto internacional entre las instituciones antes citadas. Para ello se toman como referencia los planes de estudios y prácticas organizativas de ambas instituciones y, para el estudio empírico, el "Modelo de Sistemas Dinámicos de Identidad de Roles" de Garner \& Kaplan (2018). En base al análisis cualitativo del contenido, se sigue un proceso de categorización mixta inductiva-deductiva, y codificación axial, a partir de las narraciones de los y las estudiantes. Los resultados se presentan ilustrados con extractos narrativos y organizados en tres categorías matrices y catorce subcategorías. Tras el análisis se observa cómo, a lo largo de las prácticas, las dimensiones de lo personal y lo profesional configuran un binomio de evidencias de desarrollo identitario que se solapan y retroalimentan, proceso en el cual la dinámica transnacional genera contraste y autorreflexión.

PALABRAS CLAVE: identidad docente; intercambio académico; prácticum; perfiles profesionales. 


\title{
"Looking in the mirror": the construction of professional identity as a teacher during school placements
}

\begin{abstract}
The construction of professional identity occupies a complex place among the various components of effective teacher training. School placements, in particular, represent fundamental sites for the identity development of the student, with oftentimes notable and distinctive differences apparent according to the institutional context in which this takes place. This article compares aspects of school placements within Primary Education and Early Childhood degrees in Spain and England, taking the universities of Girona and Gloucestershire as examples. We go onto present the results of a study on the construction of teacher identity with a group of second-year undergraduate students $(\mathrm{N}=16)$ participating in a transnational project across the two universities. For this, we look across the curricula and organisational practices of both institutions, drawing on Garner \& Kaplan's (2018) "Model of Dynamic Role Identity Systems" for the empirical study. We apply qualitative content analysis, a mixed inductive-deductive categorisation technique, and axial coding to an analysis of the students' narratives and provide illustrative examples of these. The data analysed are presented in three matrix categories and 14 subcategories, that highlight the dynamics of identity development, as seen and exemplified among the students of both nationalities. We observe how the transnational dynamic feeds the contrast and self-reflection and how the personal and the professional form a binomial of these dimensions that spill-over, one to the other.
\end{abstract}

KEYWORDS: teacher identity; academic exchange; school placement; professional profiles.

\section{Introducción}

A partir de unas directrices generales de ámbito nacional sobre el prácticum en los grados de magisterio (Orden Ministerial ECI/3857/2007 de 27 de diciembre, publicada en el BOE núm. 312 de 29 de diciembre de 2007), cada universidad española determina en su programa de estudios los momentos y concreciones en referencia a las prácticas externas de sus alumnos. En esta singularización, emergen importantes elementos diferenciales entre instituciones, que Lorenzo (2010) ha acotado a los componentes culturales, científicos, pedagógicos y prácticos. Algo semejante sucede en el Reino Unido, donde la regulación ejercida a nivel nacional por el Department for Education (DfE) establece unas pautas de obligado seguimiento (actualmente nombradas ITT Core Content Framework (ITTCCF), de 2019) que también, localmente, cada universidad adapta y encaja a su programa, contexto, cultura y tradición formativa. 
Los periodos de prácticas externas de los futuros maestros son valorados, en general, por todos los agentes implicados (alumnos, tutores y mentores), como momentos clave para la formación inicial del docente, y, en definitiva, para el desarrollo de una identidad profesional en construcción (Esteve et al., 2010, Jarauta \& Pérez, 2017). Se trata de un tema, el identitario, a la vez que crucial, de difícil conceptualización, pues, en general, la identidad profesional del maestro se presenta como un proceso complejo y dinámico en continuo desarrollo, desde la formación inicial hasta la veteranía (Beijaard, 2019; Cantón \& Tardif, 2018; Carrillo \& Flores, 2018). Siendo las competencias docentes (teaching standards) habilitadoras para el ejercicio de la profesión uno de los constituyentes identitarios permanentemente sujetos a debate y discusión (Callealta et al., 2020).

A su vez, podría afirmarse que la construcción de la identidad profesional ocupa uno de los dominios más abstractos y complejos entre los componentes (contenidos, objetivos y funciones) de la formación docente, moldeado por procesos reflexivos y de contraste (por ejemplo, entre teoría y práctica). Sin olvidar, así mismo, la intervención e influencia decisiva de la figura del experto formador/tutor/mentor, en la configuración de lo que McIntyre \& Hobson, (2016) han denominado "tercer espacio" del desarrollo identitario del docente en formación (en alineación con la teoría del tercer espacio, atribuida al académico de Harvard Homi K. Bhabha). Cabe considerar, también, que en el proceso identitario se incorporan, simultáneamente, aspectos propios de la profesión y aquellos de índole claramente personal, tales como las creencias, ideales o proyectos de vida de los propios y las propias estudiantes. Carrillo y Flores (2018), añaden a la dimensión personal y la dimensión profesional, la dimensión situacional, constituida por la comunidad educativa en la que se desarrolla la labor docente (alumnos, familias, compañeros, etc.), como constituyentes identitarios del trayecto profesional. Con todo, de las muchas aproximaciones e intentos de definición recabadas y publicadas sobre la conceptualización de la identidad docente, aquello que posiblemente aglutine más consenso sea su dimensión multifacética, su naturaleza claramente dinámica, y su vinculación (necesaria e implícita) con los fundamentos mismos de la formación inicial docente (Beauchamp \& Thomas, 2009). Hanna et al. (2019), explorando la literatura con enfoques psicométricos, de naturaleza cuantitativa, han recabado evidencias sobre la relación de la identidad docente con seis áreas de desarrollo profesional, a saber: la autoimagen, la motivación, el compromiso, la autoeficacia, la percepción de la tarea y la satisfacción laboral.

Una de las aportaciones más completas e inspiradas en este campo es, a nuestro entender, la de Kapplan y Garner (2017), en su modelo de "Sistemas Dinámicos de Identidad de Roles", aplicado de forma exhaustiva y ejemplificadora a un caso de identidad docente en Garner y Kapplan (2018), donde se concibe el aprendizaje/formación del maestro como un proceso contextualizado y dinámico desde la integración de los componentes culturales, de planificación, del contexto social, y del dominio o ámbito de acción profesional [ver Figura 1]. 


\section{Figura 1}

Modelo de Sistemas Dinámicos de Identidad de Roles

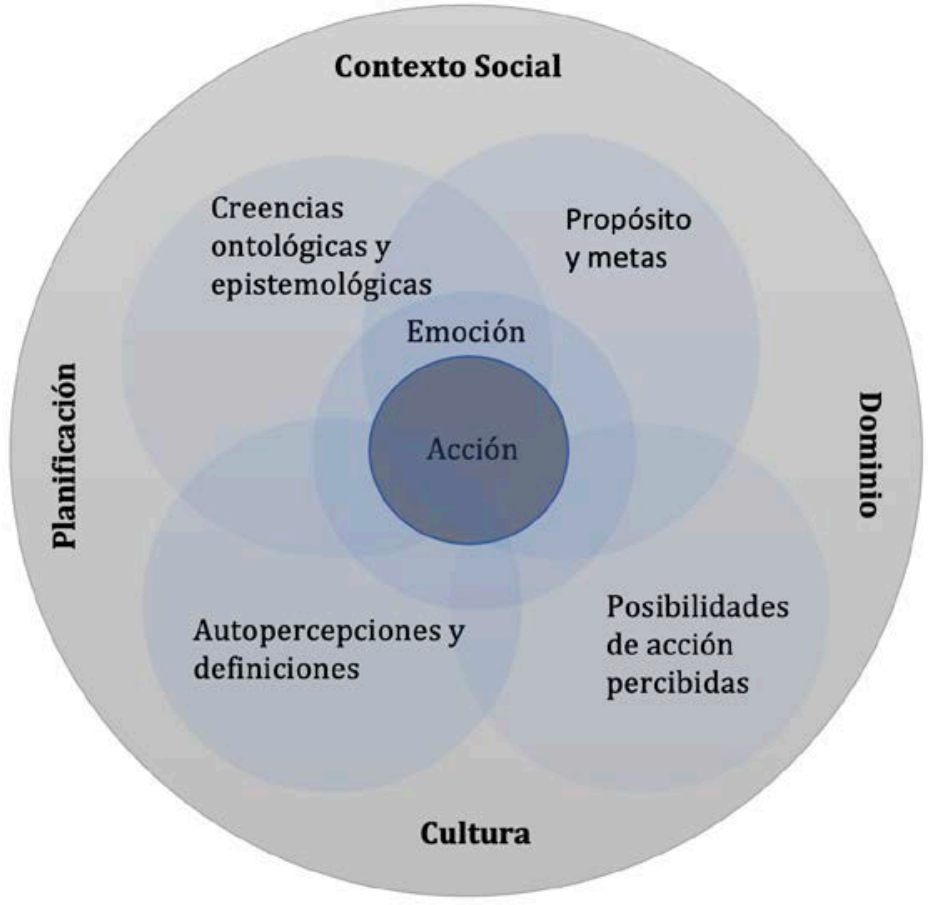

Nota. Adaptación de los autores al español del diagrama sobre el constructo "Dynamic Systems Model of Role Identity" (DSMRI) publicado en Garner y Kaplan (2018).

Entre los estudios recientes, también debemos referirnos a los resultados de Alsina et al. (2019), quienes resiguen los elementos del proceso formativo (bajo el paradigma de las "marcas de autoregulación") que intervienen en la creación del perfil e identidad profesional del maestro en formación, analizando procesos de deconstrucción, coconstrucción y reconstrucción reflejados en las producciones reflexivas del alumno en diversos contextos académicos, entre ellos el prácticum, identificando hasta doce categorías de análisis, por ejemplo, la "interacción con el contexto (centro escolar)". Por su parte, Falcón \& Arraiz (2020) realizan un estudio comparativo de relatos, obtenidos de diferentes grupos de contraste entre alumnos y alumnas de magisterio, cuyos hallazgos más relevantes inciden en la necesidad de la participación del alumno en la definición de su perfil profesional, junto a la importancia del acompañamiento y la disposición de herramientas crítico-reflexivas generadoras de (auto)narrativas (diarios personales, ePortafolio, entre otros). Lamote \& Engels (2010) observan, en un estudio de caso, una multiplicidad de rasgos y perfiles asociados a otras tantas variables, destacando las relativas al género y, también, las asociadas a la carga de experiencia práctica acumulada por el futuro docente.

Así mismo, las dinámicas de aprendizaje vicario entre iguales -es decir, el aprender de la experiencia del otro-, son fruto de procesos de intercambio (co)activo, que 
implican la (co)construcción e interacción discursiva entre los implicados, bien entre individuos, bien desde la configuración de equipos de trabajo bajo relaciones de reciprocidad (Myers, 2018, 2020). Centrándonos en experiencias de formación inicial docente, resultados como los expuestos en Steenekamp et al. (2018), a propósito de actividades experienciales planteadas fuera del campus universitario, realzan la influencia de la instrucción vicaria entre iguales en la (re)definición del perfil profesional docente. También, cuando es el caso, si los procesos son coparticipados desde, y a través, de herramientas de comunicación digital y prácticas colaborativas en la línea (Grion \& Varisco, 2007; Munday et al., 2017).

En este artículo nos interesa, en primer lugar, exponer y comparar los ejes principales sobre los que se programan y ejecutan las prácticas externas en el contexto universitario español e inglés a través de los casos de las universidades de Girona y Gloucestershire, señalando similitudes y diferencias entre los dos enfoques y realidades, pero, también, destacando problemáticas y aspectos críticos planteados en cada caso. En segundo lugar, se orienta el foco del estudio hacia la construcción de la identidad profesional de los maestros en formación, para lo cual se realiza un trabajo empírico desde datos experienciales recopilados en un proyecto transnacional con estudiantado de ambas universidades, realizado a lo largo del curso 2018-2019. Esto es, justo antes del desenlace del Brexit y de la pandemia del Covid-19, dos sucesos que han vuelto a levantar fronteras entre naciones y puesto en evidencia los retos y las fragilidades del entendimiento y la cooperación internacional.

\section{Método}

\section{Muestra y contexto de la investigación}

La muestra del estudio la constituyen dieciséis alumnos y alumnas de segundo año, reclutados tras una llamada generalizada y selección con entrevista a los interesados. Tras lo cual, se escogieron ocho participantes de cada una de las universidades, en Girona y Gloucestershire, siendo mayoritarias las mujeres (14) respecto los varones (2), en consonancia con la tendencia del alumnado de estos grados. La edad media del grupo era de entre 20 y 21 años. Así mismo, de los estudiantes españoles, siete alumnas cursaban el Doble Grado de Educación Infantil y Primaria, y un alumno el Grado de Primaria. Por su parte, seis alumnas inglesas cursaban estudios similares al Grado de Educación Primaria (Bachelor of Education, BEd) y dos alumnos un grado similar al Grado de Educación Infantil (Early Childhood Studies, BA) [ver Tabla 1].

\section{Tabla 1}

Datos identificativos de los participantes ordenados en parejas transnacionales

\begin{tabular}{lllll}
\hline ALUMNO & UNIVERSIDAD & \multicolumn{1}{c}{ GRADO } & GÉNERO & MENCIÓN \\
\hline EST1/U1 & Girona & Doble grado EI/EP & F & TIC $(*)$ \\
EST1/U2 & Gloucestershire & Bachelor of Education BEd & F & MFL $(* *)$
\end{tabular}




\begin{tabular}{lllll} 
EST2/U1 & Girona & Doble grado EI/EP & $\mathrm{F}$ & Inglés \\
EST2/U2 & Gloucestershire & Bachelor of Education BEd & $\mathrm{F}$ & MFL \\
EST3/U1 & Girona & Doble grado EI/EP & $\mathrm{F}$ & TIC \\
EST3/U2 & Gloucestershire & BA Early Childhood Studies & $\mathrm{F}$ & - \\
EST4/U1 & Girona & Doble grado EI/EP & $\mathrm{F}$ & Artes \\
EST4/U2 & Gloucestershire & BA Early Childhood Studies & $\mathrm{M}$ & - \\
EST5/U1 & Girona & Doble grado EI/EP & $\mathrm{F}$ & Inglés \\
EST5/U2 & Gloucestershire & Bachelor of Education BEd & $\mathrm{F}$ & MFL \\
EST6/U1 & Girona & Doble grado EI/EP & $\mathrm{F}$ & Inglés \\
EST6/U2 & Gloucestershire & Bachelor of Education BEd & $\mathrm{F}$ & MFL \\
EST7/U1 & Girona & Doble grado EI/EP & $\mathrm{F}$ & Ciencias \\
EST7/U2 & Gloucestershire & Bachelor of Education BEd & $\mathrm{F}$ & MFL \\
EST8/U1 & Girona & Educación Primaria & $\mathrm{M}$ & Música \\
EST8/U2 & Gloucestershire & Bachelor of Education $B E d$ & $\mathrm{~F}$ & MFL \\
\hline
\end{tabular}

Notas. (*) Tecnologías de la Información y la Comunicación. (**) Modern Foreign Languages

El proyecto se diseñó y realizó a lo largo del curso académico 2018-2019, e incluyó una estancia de varios días de todos los participantes en cada una de las instituciones, la primera de ellas en Gloucestershire a finales del primer semestre, donde se acordaron las parejas y se co-diseñó el proyecto en Elearn (entorno virtual de aprendizaje de la Universidad de Gloucestershire utilizado para los cursos y proyectos), entre otras actividades culturales y académicas, incluyendo la visita a una escuela receptora de practicantes situada en Gales, con sistema educativo bilingüe. Antes y después del segundo encuentro presencial, esta vez en Girona, a principios de mayo, con un programa cultural y académico parecido al primero, los alumnos de ambas universidades realizaron estadías de prácticas externas en la modalidad de alternancia académica. Momento en el cual los y las estudiantes produjeron las narraciones de tipo descriptivas, reflexivas, y dialógicas (con su semejante), que sustentan el estudio.

\section{Hipótesis y objetivos}

El trabajo parte de la hipótesis que la identidad y perfil profesional de los maestros en formación se reconstruye y desarrolla sustancialmente durante los periodos de prácticas docentes externas. En base a esto, y con las herramientas y procesos descritos en este apartado, se proponen estos dos objetivos.

1. Describir y valorar las dinámicas entre iguales y los resultados del aprendizaje vicario (de la experiencia ajena) en parejas de alumnos transnacionales, utilizando para ello una plataforma de intercambio narrativo en línea.

2. Observar y analizar qué aspectos experienciales de las prácticas externas de los maestros en formación intervienen en la (re)construcción de su perfil profesional y su desarrollo identitario. 


\section{Diseño y procedimiento}

En la primera parte del estudio, se han analizado los datos contenidos en los textos relacionados con el prácticum, planteando un estudio bajo el paradigma de la investigación documental, caracterizada, como describe Gómez (2011), por el enfoque interpretativo y, en nuestro caso, de índole comparativo. Para ello se ha procedido, pues, a la recopilación de los documentos fundamentales que regulan las prácticas en ambos contextos formativos. Es decir, los de tipo legislativos y, más particularmente, los que reflejan y concretan las adaptaciones particulares de ambas instituciones, nos referimos aquí a las guías de prácticas (o del prácticum) en España, o el placement guidance en Inglaterra. Estos documentos se han triangulado con dos otras fuentes primarias, a saber, sendas entrevistas con los coordinadores de prácticas externas en Girona y Gloucestershire, y, finalmente, con nuestra propia experiencia de campo como formadores-tutores en el ámbito de educación (véase un estudio semejante sobre el prácticum, de tipo comparativo, entre instituciones de Brasil, Colombia y España, en Madalena et al., 2020).

Para el estudio sobre la construcción identitaria de los futuros maestros, se recurre a un análisis del contenido cualitativo de textos producidos por los y las estudiantes durante las prácticas escolares, aplicando el desarrollo de categorías mixtas, inductivas-deductivas (Andréu, 2002), con soporte del software ATLAS.ti (.8). Las narraciones a las que nos referimos tienen a su vez un componente dialógico, puesto que se construyeron por parejas (transnacionales) de alumnos, que intercambiaron y comentaron sus experiencias durante los respectivos periodos del prácticum, a partir de un guion temático orientativo facilitado por los tutores-investigadores. Para ello se utilizó una plataforma en línea (Elearn) en la que se habilitaron ocho espacios de conversación (chats) de acceso restringido, uno por pareja. Los alumnos autorizaron protocolariamente el uso y divulgación anonimizada de los datos con finalidades de investigación educativa.

El estudio de narrativas en educación se ha desarrollado notoriamente desde finales del siglo XX, habiendo alcanzado, en la actualidad, un estatus cualificado en la profundización de los "saberes experienciales", individuales o colectivos (DeloryMomberger, 2014). Teniendo en mente el perfil de los maestros y las maestras en formación, objeto de nuestra atención, así como el objetivo de indagación entorno a la construcción de identidades, reconocemos como propias las siguientes apreciaciones que, a su vez, dan sentido a la expresión "mirarse al espejo" del título de nuestro artículo:

La narración, por lo tanto, permite a cada sujeto entrar en comunicación no solo con los demás, sino consigo mismo, y en este sentido es un instrumento excelente para una reproyección existencial, individual y social a partir de la reflexión sobre sus propias experiencias y vivencias, del redescubrimiento de sus propios valores y del reconocimiento de la unicidad de su historia de vida. (Rodríguez Illara \& Anaccontini, 2019,13)

En el estudio de las narrativas se ha aplicado el paradigma del análisis de datos cualitativo (Silverman, 2014), escogiendo la categorización deductiva para obtener las 
categorías matrices (cotejando los datos con la literatura de referencia para los investigadores) y un proceso inductivo para extraer y determinar las subcategorías asociadas en cada caso. El concreto el trabajo se ha secuenciado en las siguientes fases:

1. Una primera lectura independiente de los textos producidos por las parejas de alumnos a cargo de ambos tutores-investigadores en Girona y Gloucestershire. Los textos extraídos de la plataforma de comunicación en línea los constituían un total de ocho documentos de texto (uno por pareja transnacional), sumando en total de 19.189 palabras, de las cuales el 54,13\% correspondía a intervenciones de los alumnos españoles y el 45,87\% de los ingleses. En esta lectura cada investigador, siguiendo un proceso de codificación abierta, y una comparación entre los diferentes casos (parejas) que componían la muestra, elaboró una propuesta de categorías preliminar sustentada, en su conjunto, por un total de 318 unidades de contenido.

2. Los análisis individuales se discutieron conjuntamente y redefinieron por un proceso de reducción, ayudados en este punto por la triangulación con referencias significativas en el campo, en particular, adoptando como principal referente el modelo de sistemas dinámico (DSRMI) desarrollado por Kaplan \& Garner (2017). Tras lo cual, se crearon un total de tres agrupaciones temáticas (categorías macro) con sus memos (descripciones, preguntas e hipótesis) correspondientes, en concreto: a) expectativas iniciales y autoimagen; b) confrontaciones con el contexto profesional, c) interlocuciones y contraste entre iguales.

3. Los datos seleccionados se reorganizaron, con un trabajo estrictamente analítico de codificación axial, dando consistencia a la relación entre categorías y subcategorías, tras el procesamiento y reducción de las unidades de contenido. Ello permitió una asignación definitiva de las subcategorías de forma fundamentada y enriquecida por la condensación de significados (códigos) que concentran ideas, creencias, valores y percepciones, conocido también como proceso de saturación (Saldana, 2016). Estas tres categorías temáticas, junto a las subcategorías generadas y agrupadas a su entorno, se explican y ejemplarizan en el apartado de resultados.

\section{Resultados}

\section{Prácticas escolares en UdG y UoGL}

El estudio comparativo de las prácticas externas en las universidades de Girona y Gloucestershire se ha organizado de modo que se facilite el ejercicio de contraste entre los datos seleccionados, escogiendo para ello aquellos aspectos considerados clave en la configuración del prácticum. Para ello presentamos en las tres tablas que siguen (Tablas 2, 3 y 4), la información referida a estos tres ámbitos: 1) Marco curricular y organizativo; 3) Desarrollo competencial y evaluación; 3) Seguimiento del alumno y relación intercentros. 


\section{Tabla 2}

Marco curricular y organizativo

\begin{tabular}{|c|c|c|}
\hline Ámbito & UdG & UoGL \\
\hline $\begin{array}{l}\text { Organización de } \\
\text { los Grados de } \\
\text { formación } \\
\text { docente } \\
\text { (programas, } \\
\text { itinerarios, } \\
\text { cualificaciones...) }\end{array}$ & $\begin{array}{l}\text { - Ocho menciones o itinerarios de } \\
\text { especialización disciplinar (21 ECTS } \\
\text { en materias optativas + } 10 \text { ECTS del } \\
\text { Prácticum): } \\
\text { 1. Mención en Tecnologías de la } \\
\text { Información y la Comunicación } \\
\text { 2. Mención en Educacióń Musical } \\
\text { 3. Mención en Lengua Extranjera } \\
\text { (inglés) } \\
\text { 4. Mención en Educacióń Física } \\
\text { 5. Mención en Artes Visual y } \\
\text { Plásticas } \\
\text { 6. Mención en Educacióń } \\
\text { Científica y Ambiental } \\
\text { 7. Mención en Expresiones y } \\
\text { Ambientes a la Escuela } \\
\text { Maternal (solo Infantil) } \\
\text { 8. Mención en Teología Católica y } \\
\text { su Pedagogía }\end{array}$ & $\begin{array}{l}\text { Students can select a specialism } \\
\text { made up of } 2 \text { additional modules in a } \\
\text { foundation subject in their 2nd year } \\
\text { of study. } \\
\text { They can currently choose from } 7 \\
\text { subjects (due to change to 10): } \\
\text { 1. Primary languages } \\
\text { 2. History } \\
\text { 3. Geography } \\
\text { 4. Religious Education } \\
\text { 5. Physical Exercise } \\
\text { 6. Art } \\
\text { 7. Computing } \\
\text { Each are 15 CAT modules [2]. } \\
\text { All other modules are compulsory. } \\
\text { Bachelor of Arts (BA) (Hons) routes, } \\
\text { 3 years, at UoGL include [3]: } \\
\text {-Early Childhood Studies } \\
\text {-Education } \\
\text {-Education, Inclusion \& Special } \\
\text { Educational Needs }\end{array}$ \\
\hline $\begin{array}{l}\text { Dedicaciones } \\
\text { totales en ECTS } \\
\text { curriculares / } \\
\text { horas en el } \\
\text { centro de } \\
\text { prácticas }\end{array}$ & $\begin{array}{l}\text { - Grados de Educación Infantil y } \\
\text { Educación Primaria: } 44 \text { ECTS ( } 720 \\
\text { h), repartidos en Prácticum I } \\
\text { Prácticum II }\end{array}$ & $\begin{array}{l}\text { For all BEd routes, a minimum of } 120 \\
\text { days of placement is required over } \\
\text { the course of the } 3 \text { years. Students } \\
\text { will typically accrue 140-150 days. }\end{array}$ \\
\hline
\end{tabular}


- Doble grado Infantil y Primaria: 88 ECTS (1.440h), con dos Prácticum I y dos Prácticum II

(Además, cabe añadir las estancias de observación de $1^{\mathrm{o}}$ y $2^{\mathrm{o}}$ curso, aunque no estén contabilizadas dentro del prácticum)

\section{Ubicación de las prácticas externas a lo largo del Grado}

- 1r curso: 3 semanas $(1+2)$ de estancia en los centros (vinculadas al Módulo: "Observación sistemática y análisis de contextos")
- 2o curso: 4 semanas $(2+2)$ de estancia (Vinculadas al módulo: "Organización del espacio escolar, materiales y habilidades docentes"

- 3r curso (Infantil/Primaria/DG): 10 semanas de Prácticum I (Primer Semestre)

- 4o curso (DG): Prácticum I (bis): 9 semanas en jardín de infancia.

- 4o curso (Infantil/Primaria): 13 semanas de Prácticum II (Segundo Semestre)

- 5o curso (DG): 24 semanas

$(12+12)$ para realizar el Prácticum

II de Infantil y Primaria (en el mismo centro escolar)
For the BA (Hons) Early Childhood Studies route, a total of 6 weeks placement is required in each year. For BA Education and BA Education, Inclusion \& Special Educational Needs, placement is generally followed but is optional.

1st Year (L4) 7-week placement- 1 module - 15 CATS, can be split across two settings -2 blocks of time.

2nd Year (L5) 10-week placement- to be split across 2 settings. 2 modules 30 CATS in total

3rd Year (L6) (9-week placement) 1 setting only (cannot be spilt across 2 settings) - 30 CATS - 1 block of time

Placements are in blocks in order to show sustained classroom practice over time.

Notas. [1] BEd with Qualified Teacher Status (QTS) means the student is eligible to teach in all schools in England and Wales. [2] Under the Credit Accumulation and Transfer (CAT) Scheme, each year of a full-time undergraduate degree programme equates to 120 credit points. 120 CAT points equates to 60 ECTS credits. [3] BA \& BSc (Hons) routes (some of which can be followed part-time) are first degrees without QTS. Students following these routes can also seek to go into the teaching profession. All non-BEd students wishing to gain QTS must follow a 1year Postgraduate Certificate in Education (PGCE). Those wishing to gain Early Years Teacher Status (EYTS) need to take: Early Years Initial Teacher Training (EYITT) 1 year.

Los comentarios sobre la tabla 2 deben empezar por señalar unas diferencias sustanciales en la configuración de las titulaciones en uno y otro país, hecho explicable con la separación, en España, a todos los efectos, de los estudios de Pedagogía respecto de los de Magisterio. Debe observarse también la duración de los programas, de cuatro años en España y tres en Reino Unido, y una mayor diversidad de opciones de especialización, en Gloucestershire, enfocadas a diferentes franjas de edad de los alumnos. En ambos casos se puede optar a la especialización disciplinar, y se ofrece un modelo de "Prácticum en alternancia", es decir, combinando a lo largo del grado la formación en la universidad y las prácticas externas, característica importante en la que las dos instituciones convergen de forma clara. 


\section{Tabla 3}

Desarrollo competencial y evaluación

\begin{tabular}{lll}
\hline \multicolumn{1}{c}{ Ámbito } & \multicolumn{1}{c}{ UdG } & \multicolumn{1}{c}{ UoGL } \\
\hline $\begin{array}{l}\text { Competencias } \\
\text { profesionales }\end{array}$ & Agrupadas en tres ámbitos o áreas & The Teachers' Standards (DfE, \\
asociadas al & concretan un conjunto de trece & 2013) used in the UK are made up of \\
Prácticum & two set areas: (i) the first around \\
& competencias extraídas de la & teaching; (ii) the second on personal \\
& Memoria del Grado, a su vez & and professional standards, which is \\
& definidas por un listado de & essentially a broad statement about \\
& subcompetencias o indicadores & how teachers are expected to \\
& competenciales, en este caso & conduct themselves. ${ }^{1}$
\end{tabular}

específicos del Prácticum. Las tres

áreas son:

1."Actuación profesional en el aula"

2. “Colaboración en el marco institucional escolar y de la comunidad educativa"

3. “Reflexión, investigación e innovación docente".

The 8 Teaching Standards in Part 1:

1. High Expectations

2. How Pupils Learn

3. Subject and Curriculum

4. Classroom Practice

5. Adaptive Teaching

6. Assessment

7. Managing Behaviour

8. Professional Behaviours

- School-based mentor:

\section{Seguimiento y evaluación del estudiante}

- Mentoría desde la escuela:
La denominación de "mentor o mentora" se implanta el curso 20192020. Tutor o tutora de aula que está al cargo de la supervisión y evaluación del practicante, para lo cual dispone de una rúbrica de evaluación. En el prácticum ligado a mención, la mentoría recae en el o la especialista, habitualmente sin tutoría.

- Tutoría desde la facultad: Docente en los estudios que realiza el seguimiento del alumno a través de un mínimo de cuatro tutorías pautadas en el calendario de prácticas, más un mínimo de una visita a la escuela, para seguimiento,
Class teacher or another member of staff overseeing student experience. Observes practice, provides feedback and support with target setting, writes reports at end of each placement. Provides weekly professional discussions during placement.

\section{- Link tutor:}

Quality assurance role. Provides 2 visits:

-First, checking in, making sure roles and expectations are understood and being met.

-Second, a review of progress and validation of the schools'

judgements and outcomes. Would

1 The Teachers' Standards can be found on the GOV.UK website: https://www.gov.uk/government/publications/teachers-standards 
observación y evaluación del estudiante.

- Coordinadores de Prácticum: gura de gestión, una en la facultad y ra en cada una de las escuelas laboradoras. normally be in school with some observations of the students' teaching.

- Placement coordinators:

Collates offers and matches students to the schools. Oversees a central records system.

En la tabla 3, debemos comentar, de entrada, un marco conceptual convergente entre ambas instituciones, tras el paso al currículum competencial en España, tras la última reforma universitaria ("Proceso de Bolonia"). Como diferencias destacadas, debe notarse el valor de calificación profesional de los Standards ingleses, que habilitan directamente para el ejercicio de la profesión (no existen las oposiciones), y la implicación en su definición, supervisión y evaluación del Departamento de Educación estatal (DfE). La reciente adopción del término "mentor/a" en Cataluña, a petición del Departamento de Educación autonómico, debe interpretarse como una clara influencia del sistema educativo inglés.

\section{Tabla 4}

Documentación y relación intercentros

\begin{tabular}{|c|c|c|}
\hline Ámbito & UdG & UoGL \\
\hline \multirow[t]{2}{*}{$\begin{array}{l}\text { Documentos de } \\
\text { soporte para el } \\
\text { alumno }\end{array}$} & $\begin{array}{l}\text { Documento "Guía del Prácticum" } 2 \text { : } \\
\text { Documento marco, de acceso abierto, que } \\
\text { informa e instruye sobre todos los } \\
\text { aspectos relacionados con el prácticum, } \\
\text { revisado y actualizado cada curso con } \\
\text { concurso del equipo de tutores. }\end{array}$ & $\begin{array}{l}\text { Every placement module has } \\
\text { its own 'Placement } \\
\text { handbook'. There are also a } \\
\text { series of template forms for } \\
\text { students to use in lesson } \\
\text { planning and for capturing } \\
\text { placement reports. }\end{array}$ \\
\hline & $\begin{array}{l}\text { Dossier de Anexos (calendarios, } \\
\text { orientaciones, rúbricas, etc.) } \\
\text { Documentos prácticos que van a utilizar } \\
\text { todos los actores en diversos momentos } \\
\text { de las prácticas. }\end{array}$ & $\begin{array}{l}\text { These materials are shared } \\
\text { with schools through the } \\
\text { University Elearn platform. } \\
\text { Partnership agreements with } \\
\text { schools have to be read and } \\
\text { signed before a School can } \\
\text { host a student. }\end{array}$ \\
\hline
\end{tabular}

2https://www.udg.edu/ca/portals/42/OpenContent/Files/10487/GUIA_PRACTICUM_MESTRES_20202021 def.pdf

${ }^{3}$ https://www.udg.edu/ca/portals/42/OpenContent/Files/10487/Annexos practicum 20-21 def6.pdf 


Relación entre
universidad y
centros de
prácticas
prácticas
Un "técnico del prácticum" contratado desde la facultad se ocupa de la gestión administrativa de asignación de estudiantes y comunicación con los centros

El "coordinador académico de prácticum" en la facultad: responsable de gestionar y aplicar las directrices y mantener la comunicación con alumnos y tutores.

Coordinador del Prácticum en los centros externos: supervisa los procesos de asignación y acogida.

Comisión de Prácticum en la que interviene la administración educativa del gobierno autonómico, realiza generalmente una reunión anual de seguimiento.
The Primary Partnerships lead oversees placements, ensures there are sufficient settings, communicates with schools, recruits schools to the partnership. Links to the partnership are strong, providing support for mentors and link tutors especially in relation to monitoring and tracking of student progress. They determine if a student requires intervention between placements.

A primary partnerships board meets 3 times a year to review practice and make sure training reflects what is current in schools.

En la tabla 4 se observa una estructura de gestión semejante o muy equiparable en una y otra universidad, hecho que responde a una necesidad de comunicación y coordinación entre instituciones. No obstante, del estudio documental se desprende e interpreta una mayor interconexión y voluntad de vínculo entre centros formativos (facultad-escuelas) en el modelo de Gloucestershire, contemplándose aquí la participación de los mentores en actividades académicas de la facultad, siendo este uno de los aspectos críticos y con necesidad de revisión entre las dinámicas organizativas observadas en Girona (para ampliar la información sobre el modelo de prácticum en la Universidad de Girona, véase: Masgrau-Juanola et al., 2017).

\section{Estudio empírico: identidad(es) en construcción}

Se presentan los hallazgos del estudio organizando la información en las tres categorías matrices, a las que se asocian las subcategorías generadas tras el análisis de los datos (Tablas 5, 6 y 7). Se aporta, en cada caso, un par de citas obtenidas de la misma pareja, lo cual va variando en cada apartado en aras de la representatividad y consistencia de los datos.

\section{Expectativas iniciales y autoimagen}

Hace referencia a manifestaciones sobre qué se espera de las prácticas, a cómo se encaran desde el inicio, y sobre cómo se ve el alumno a sí mismo y cómo lo ven los demás. 


\section{Tabla 5}

Categoría 1: Expectativas iniciales y autoimagen

\begin{tabular}{ll}
\hline Subcategorías & \multicolumn{1}{c}{ Citas de las narrativas } \\
\hline $\begin{array}{l}\text { Prejuicios } \\
\text { experienciales }\end{array}$ & Miro de aprender mucho en estas prácticas porque no estoy segura de \\
& si voy a repetir semejante experiencia con un grupo tan bueno, una \\
& buena mentora y un equipo tan innovador. (EST3/U1) \\
& En mi opinión y experiencia, tener un buen mentor en tus prácticas es \\
& crucial. (EST3/U2) \\
Certezas y valores & Cuando empecé el Grado estaba totalmente convencida de mi \\
& preferencia por enseñar en Primaria por el nivel de las materias. En \\
& cambio, ahora tengo mis dudas. (EST 2/U1) \\
& Te puedo decir que, después de algunas experiencias con los más \\
& pequeños, encontré que seguramente podría trabajar en infantil \\
& (EST2/U2) \\
Temor al fracaso & Al principio estaba muy preocupada debido a un alumno que es muy \\
& nervioso y acostumbra a distorsionar la clase. (EST1/U1) \\
& Estaba algo intranquila, pero me dije a mi misma que era la primera vez \\
& que iba a ser observada, que solo querían revisar mi progreso actual. \\
Idealización & EST1/U2) \\
profesional & La escuela es un centro moderno, con maestros jóvenes que están \\
& realmente implicados e interesados, siempre buscando como beneficiar \\
a los alumnos. (EST4/U1) \\
Construyendo relaciones entre padres, niños, compañeros, y con todo el \\
Puturo \\
resto, se puede desarrollar una sociedad más fuerte y cohesionada. \\
(EST4/U2) \\
Quería un centro con niños de diferentes países para ver las dificultades \\
y desafíos de este tipo de escuelas, en caso de que cuando sea maestra \\
tenga que trabajar en una parecida. (EST1/U1) \\
Sería un verdadero reto trabajar en una escuela con tanta diversidad \\
de idiomas entre los alumnos (EST1/U2)
\end{tabular}

Es una categoría muy nutrida de evidencias, hecho que refleja el interés y la importancia que se otorga a las prácticas externas por parte de los futuros maestros y maestras. Por lo general, estos son conscientes de que van a presentarse situaciones en las que van a cuestionarles o, por lo contrario, reforzarles algunos de sus principales fundamentos identitarios (sentido vocacional, modelo docente, registro comunicativo...).

\section{Confrontaciones en el contexto profesional}

Esta categoría aglutina binomios sobre los cuales el estudiante reflexiona a menudo a lo largo de sus prácticas, son elementos a veces presentados como contrarios o en conflicto, cuestiones que toman relieve y encuentran oportunidades de reequilibrio gracias a la inmersión en el contexto profesional real. 


\section{Tabla 6}

Categoría 2: Confrontaciones en el contexto profesional

\begin{tabular}{ll}
\hline Subcategorías & \multicolumn{1}{c}{ Citas de las narrativas } \\
\hline Teoría y práctica & $\begin{array}{l}\text { Después de este último tiempo en la universidad, aprendiendo cosas } \\
\text { interesantes sobre cómo ser más efectiva enseñando a los niños, me } \\
\text { propongo interactuar más con ellos y aprovechar las oportunidades que } \\
\text { se me presenten. (EST5/U1) }\end{array}$
\end{tabular}

En nuestras prácticas a menudo nos encargan completar en las escuelas aquello que hemos estado aprendiendo en la universidad. (EST5/U2)

Observar y actuar Me doy cuenta de que ahora me implico más en las actividades de la clase, mi mentor me deja tomar parte y se me trata como a un maestro más. (EST5/U1)

A menudo siento lo mismo cuando estoy en el prácticum, necesito unos cuantos días para tomar decisiones y mostrar una buena iniciativa. (EST5/U2)

\section{Identidad y diversidad}

Emoción y razón

Autopercepción de progreso (el "antes y ahora")
Debería haber una mayor presencia masculina en las primeras edades y no eso de que solo "las mujeres trabajan con niños", pues no es, ni debería ser el caso. (EST4/U2)

Todos los niños son diferentes y especiales, y he aprendido que cada uno de ellos tiene sus necesidades y reclama su atención. (EXT4/U1)

Fue fantástico para mi porqué pude aprender algo sobre los niños y sobre aquello que les interesa. (EST7/U2)

Me siento tan bien cuando estoy en la escuela, cuando doy la clase, con la ayuda de mi mentora, porque pienso que es esto lo que siempre he querido ser. (EST7/U1)

El tiempo ha volado durante estas prácticas. Han sido las dos semanas más productivas de todo el año. Durante estos días he aprendido innumerables valores, metodologías y he crecido como maestro y como persona. (EST8/U1)

Yo siento lo mismo cuando voy a la escuela. Pretendo crecer como maestro, pero acabo cambiando igual o más como persona. (EST8/U2)

La riqueza formativa de las prácticas escolares se pone de manifiesto de forma muy explícita en esta categoría, con el foco puesto en cinco elementos de reflexión que el futuro maestro o maestra debe reasentar entre sus componentes de identidad. Las citas de la tabla 6 son expresiones ricas en evidencias sobre avances en distintas facetas cruciales, que son posibles gracias a oportunidades de confrontación en el contexto escolar y la asunción del rol profesional. La relación dialógica entre los participantes, como se comprueba, realza e impulsa estas evidencias. 


\section{Interlocuciones entre iguales}

Se trata de la categoría surgida explícitamente del componente internacional y transnacional (en parejas) de la muestra, hecho que refuerza las posibilidades de contraste y el factor comparativo. Es, así mismo, el momento en el que mejor visualizamos el concepto de autoreflejo identitario en las palabras del otro.

\section{Tabla 7}

Categoría 3: Interlocuciones entre iguales

\begin{tabular}{|c|c|}
\hline Subcategorías & Citas de las narrativas \\
\hline \multirow[t]{2}{*}{$\begin{array}{l}\text { Dialéctica sobre los } \\
\text { contenidos }\end{array}$} & $\begin{array}{l}\text { En Inglaterra también afirmamos que los niños tienen un buen } \\
\text { conocimiento y comprensión de las estructuras del lenguaje observando } \\
\text { el uso de palabras como "debajo" y "sobre". (EST7/U2) }\end{array}$ \\
\hline & $\begin{array}{l}\text { Me gusta la actividad que has preparado para PSHE en la que los niños } \\
\text { traen y presentan algún objeto especial para ellos. (EST7/U1) }\end{array}$ \\
\hline \multirow[t]{2}{*}{$\begin{array}{l}\text { Refuerzos } \\
\text { empáticos }\end{array}$} & $\begin{array}{l}\text { Estoy realmente perpleja sobre lo que me has contado, sobre la actitud } \\
\text { de "tú eres varón, no sirves para los pequeños". Encuentro que es arcaico } \\
\text { y quien piense así no sirve para maestro. (EST4/U1) }\end{array}$ \\
\hline & $\begin{array}{l}\text { Es fantástico oír que has aprendido de la experiencia y conocer tus } \\
\text { conclusiones. Me gusta saber que has podido observar diferentes tipos de } \\
\text { metodologías docentes y has podido decidir aquella que te gustaría } \\
\text { adoptar. (EST4/U2) }\end{array}$ \\
\hline \multirow[t]{2}{*}{$\begin{array}{l}\text { Refuerzos por } \\
\text { contraste }\end{array}$} & $\begin{array}{l}\text { ¿Que interesante! En mis prácticas nunca he tenido oportunidad de } \\
\text { discutir y plantear diferentes estrategias para gestionar la conducta de } \\
\text { los alumnos durante los cambios de clase. (EST1/U1) }\end{array}$ \\
\hline & $\begin{array}{l}\text { Debe ser realmente un reto trabajar en escuelas donde hay tal diversidad } \\
\text { de lenguas. Mi experiencia ha sido en centros con muy pocos niños de } \\
\text { habla no inglesa. (EST1/U2) }\end{array}$ \\
\hline \multirow[t]{2}{*}{$\begin{array}{l}\text { Autoreflejos } \\
\text { identitarios }\end{array}$} & $\begin{array}{l}\text { Entiendo a qué te refieres con lo de la añoranza. En mis últimas prácticas } \\
\text { tuve que regresar al centro a la semana siguiente de terminarlas porque } \\
\text { lo extrañaba demasiado. (EST2/U2) }\end{array}$ \\
\hline & $\begin{array}{l}\text { He aprendido mucho de mi mentora porque tiene una forma muy } \\
\text { especial de dar ánimos e improvisar actividades y juegos. (EST2/U2) }\end{array}$ \\
\hline
\end{tabular}

Nota. PSHE: Educación Personal, Social, Sanitaria y Económica

Se trata de una categoría con una dimensión primordialmente dialógica, siendo este un componente esencial para entender y valorar las cuatro subcategorías que la integran. En primer lugar, en la comparación entre culturas educativas sobre el eje de los contenidos (pero igualmente sobre métodos, currículum, organización, etc.) que genera, a su vez, signos identitarios en ambos interlocutores. Como se aprecia en los textos de la tabla 7, la categoría está "alimentada" por un fuerte componente empático, bien actuando como refuerzo por reafirmación de experiencias positivas, contrastando matices y diferencias en las experiencias formativas de ambos, o en reinterpretaciones de las narraciones, que provocan una "visión amplificada" del contenido referido. 


\section{Discusión y conclusiones}

Como apunta Esteve (2006), el proceso de homogeneización de la formación docente en el ámbito de los países desarrollados ha seguido un proceso imparable desde finales del siglo $\mathrm{XX}$, hecho matizado, entre otros aspectos, por el grado de centralización estatal o de autonomía de los centros formadores. El ejercicio comparativo entre las prácticas externas de futuros maestros, en una universidad española y otra de inglesa, ha dado resultados interesantes en cuanto a similitudes y contrastes respecto a la cultura formativa y organizativa de una y otra institución. Por extensión, también, aunque con cautela, se presenta la posibilidad de generalizar los resultados de la comparativa atendiendo a características nacionales, en consonancia con Egido (2020), el cual señala tres grandes tendencias con relación a la formación, acreditación, y paso a la profesión del docente en Europa. Finalmente, toda tentación homogeneizadora debe tenerse presente que, tanto España como el Reino Unido, son países de grandes dimensiones, con una importante diversidad de realidades culturales, políticas y, por ende, administrativas. Abordando una perspectiva general de los diferentes componentes descritos comparativamente al pie de las tablas de análisis (Tablas núm. 2, 3 y 4), podemos afirmar que las prácticas y culturas formativas observadas en Girona y Gloucestershire ofrecen un amplio abanico de diversidad y de matices ligados, entre otros aspectos, a las lenguas de los participantes, sus tradiciones y sus culturas (por ejemplo: horarias, organizativas, familiares, alimentarias...), puestas en contraste, de forma práctica, en los diferentes momentos y situaciones del intercambio.

La construcción de la identidad profesional docente es, sin duda, un tema de interés en torno a la formación inicial del profesorado, estudiado en la literatura más reciente desde diferentes enfoques y perspectivas. A las propuestas y modelos ya citados en la Introducción, debemos referirnos también al texto de Lopes (2007), fundamentado en la sociología, que ahonda en las "crisis de identidad del profesorado" y la construcción de nuevas identidades que requiere la era postmoderna, vinculando los procesos de construcción de identidades profesionales con los procesos de profesionalización de los docentes. Procesos de profesionalización que arrancan, sin duda, en el caso del docente, en su formación inicial y, de manera especial, en sus prácticas escolares externas, como han puesto de relieve numerosos estudios centrados, de manera particular, en el desarrollo de las competencias profesionales (Calbó, 2009; Callealta et al., 2020; Cámara et al, 2011). Igualmente, nuestro trabajo incide en una continua y necesaria revisión sobre los procesos, las percepciones, y los resultados, en relación con la coherencia formativa entre las facultades y los centros de prácticas (véase, por ejemplo, los resultados sobre la valoración del estudiantado de Poveda et al., 2021).

En nuestra investigación, el marco de las competencias profesionales toma especial relevancia a la hora de situar y comparar los marcos académicos que circunscriben las prácticas escolares en Girona y Gloucestershire, con competencias agrupadas, en cada caso, a dos o tres grandes áreas. Las cuales incluyen, a la vez que distinguen, el desarrollo como docente y futuro profesional y las evidencias de madurez, tales como la conducta o equilibrio emocional. En línea con los hallazgos de Leeferink et al. (2019), que indagan sobre las concordancias y las divergencias en la 
narrativa de diez estudiantes sobre sus prácticas docentes, de los resultados de nuestro estudio se desprende una importante interrelación entre las vivencias formativas desde la óptica personal y desde la profesional. ¿En qué sentido? Si bien las tres categorías matrices, que se formulan al inicio del análisis, nos ubican en coordenadas de índole conductual o psicológico, en las subcategorías, y las citas que las ilustran, descubrimos una estrecha vinculación entre las referencias de índole claramente intra o interpersonal y los dominios más prosaicos de la práctica docente.

Diríamos, pues, en relación con la hipótesis de trabajo planteada en un inicio, que los procesos de reflexión y transformación acaecidos en los contextos de las prácticas externas durante la formación inicial docente son altamente reveladores para la comprensión de la construcción del perfil profesional. La dinámica comunicativa de igual a igual, entre parejas de estudiantes de instituciones distintas, pero en momentos y contextos formativos similares, se ha mostrado altamente productiva y eficaz en la generación del autoreflejo en el que observar procesos madurativos personales de cada alumno. Procesos de autoafirmación, aprendizaje, y transformación, que se vislumbran cruciales para un futuro desempeño pleno de la profesión. El factor transnacional, en resumen, ha resultado ser un elemento dinamizador y catalizador de contraste, e impulsor de la mirada al otro y del diálogo consigo mismo. Diríamos, con seguridad, que el ejercicio comparativo entre estudiantado de una y otra procedencia revela un universo identitario común y reconocible desde ambas perspectivas nacionales.

Las implicaciones de esta investigación son significativas, a nuestro entender, en relación con la valoración y herramientas de seguimiento de las prácticas externas de las instituciones al cargo de la formación inicial del profesorado. Nos referimos aquí, en especial, a aquellos ámbitos (también) competenciales ligados a la parte más íntima $\mathrm{y}$, a menudo, inescrutable del alumnado. Para ello, y para poder incidir en estos aspectos, la universidad debería dotar y promover una acción tutorial potente y personalizada. Por último, debemos referirnos a las limitaciones intrínsecas a toda investigación de tipo cualitativo y las dimensiones reducidas de la muestra. Una experiencia a mayor escala, o en un marco temporal más extenso, permitiría abordar algunos aspectos aquí sin recorrido, como el análisis diferenciado por género, según la filiación profesional de los progenitores, o la modelización proyectada por tutores y mentores, entre otros temas de interés.

\section{Agradecimientos}

Se agradece la colaboración recibida, durante la elaboración del artículo, de Andrea McGowan (Academic Subject Leader for ITE at University of Gloucestershire), Emma Howell (Primary Partnerships Lead at University of Gloucestershire) y de la Dra. Montserrat Calbó (Coordinadora de Prácticum de Magisterio en la Universidad de Girona) por sus útiles comentarios con relación a la organización de las prácticas escolares en la UoGL y la UdG.

\section{Conflicto de intereses}

Los autores declaran no tener ningún conflicto de intereses. Los financiadores no tuvieron ningún papel en el diseño del estudio; en la recopilación, análisis o interpretación de datos; en la redacción del manuscrito, o en la decisión de publicar los resultados. 


\section{Contribuciones de los autores}

Conceptualización, M.A. y A.M.; metodología, M.A.; validación, A.M.; análisis de datos, M.A. y A.M.; redacción del borrador original, M.A.; redacción, revisión y edición, M.A. y A.M.; adquisición de financiación, M.A. y A.M.

\section{Referencias}

Alsina, À., Batllori, R., Falgàs, M. y Vidal, I. (2019). Marcas de autorregulación para la construcción del perfil docente durante la formación inicial de maestros. Revista Complutense de Educación, 30(1), 55-74. https://doi.org/10.5209/RCED.55466

Andréu, J. (2002). Las técnicas de análisis de contenido: Una revisión actualizada. Sevilla: Fundación de Estudios Andaluces. Disponible en: https://cutt.ly/hlo7mPp

Beauchamp, C. y Thomas, L. (2009). Understanding teacher identity: an overview of issues in the literature and implications for teacher education. Cambridge Journal of Education, 39(2), 175-189. https://doi.org/10.1080/03057640902902252

Beijaard, D. (2019). Teacher learning as identity learning: models, practices, and topics. Teachers and Teaching, 25(1), 1-6. https://doi.org/10.1080/13540602.2019.1542871

Calbó, M. (coord.) (2009). Guia per a l'avaluació de competències en el pràcticum de mestre/a. AQU. https://tuit.cat/jGQSD

Cantón, I. y Tardif, M. (Coord) (2018). Identidad profesional docente. Narcea S. A de Ediciones.

Cámara, A., Abril, A. M., Díaz, E. M., Gutiérrez, F., Párraga, J.A., Romero, M. y Ortega, J. M. (2011). Análisis de competencias en la formación de maestros a través del Prácticum. REDU. Revista de Docencia Universitaria, 9(2), 55-70. https://doi.org/10.4995/redu.2011.6158

Callealta, L., Donoso, M. y Camuñas, N. (2020). Identidad profesional docente: la influencia de las competencias y la formación inicial de los aspirantes a profesores de Educación Secundaria. Revista de estilos de aprendizaje, 13(25), 8498. http://revistaestilosdeaprendizaje.com/article/view/1880

Carrillo, C. y Flores, M. A. (2018). Veteran teachers' identity: What does the research literature tell us? Cambridge Journal of Education, 48(5), 639-656. https://doi.org/10.1080/0305764X.2017.1394982.

Delory-Momberger, Ch. (2014). Experiencia y formación. Biografización, biograficidad y heterobiografía. Revista mexicana de investigación educativa, 19(62), 695-710. https://www.redalyc.org/pdf/140/14031461003.pdf

Egido, I. (2020). El acceso a la formación inicial docente en Europa: políticas e investigación. Revista Española de Educación Comparada, 35, 197-211. https://doi.org/10.5944/reec.35.2020.24192

Esteve, J. M. (2006). La profesión docente en Europa: perfil, tendencias y problemática. La formación inicial. Revista de Educación, 340, 19-86. https://cutt.ly/BkCZU8o

Esteve, O., Melief, K. y Alsina, À. (Eds.) (2010). Creando mi profesión. Una propuesta para el desarrollo profesional del profesorado. Octaedro. 
Falcón, C. y Arraiz, A. (2020). Construcción de identidad profesional docente durante la formación inicial como maestros. Revista Complutense De Educación, 31(3), 329-340. https://doi.org/10.5209/rced.63374

Garner, J. K. y Kaplan, A. (2018). A complex dynamic systems perspective on teacher learning and identity formation: an instrumental case. Teachers and Teaching, 25(1), 7-33. https://doi.org/10.1080/13540602.2018.1533811

Gómez, J. (2011). Un espacio para la investigación documental. Vanguardia Psicológica, 1(2), 226-233. https://tuit.cat/i7g1d

Grion, V. y Varisco, B. M. (2007). Online collaboration for building a teacher professional identity. PsychNology Journal, 5(3), 271 - 284. https://cutt.ly/RlchoAQ

Hanna, F., Oostdam, R., Severiens, S. E., y Zijlstra, B. J. (2019). Domains of teacher identity: A review of quantitative measurement instruments. Educational Research Review, 27(1), 15-27. https://doi.org/10.1016/j.edurev.2019.01.003

Jarauta, B. y Pérez, M. J. (2017). La construcción de la identidad profesional del maestro de primaria durante su formación inicial. El caso de la Universidad de Barcelona. Profesorado. Revista de currículum y formación del profesorado, 21(1), 103-122. https://recyt.fecyt.es/index.php/profesorado/article/view/58053

Kaplan, A. y Garner, K. (2017). A complex dynamic systems perspective on identity and its development: the dynamic systems model of role identity. Developmental Psychology, 5(11), 2036-2051. https://doi.apa.org/doi/10.1037/dev0000339

Lamote, L. y Engels, N. (2010). The development of student teachers' professional identity. European Journal of Teacher Education, 33(1), 3-18. https://doi.org/10.1080/02619760903457735

Leeferink, H., Koopman, M., Douwe Beijaard, D. y Schellings, G. (2019). Overarching professional identity themes in student teacher workplace learning. Teachers and Teaching, 25(1), 69-89. https://doi.org/10.1080/13540602.2018.1527762

Lopes, A. (2007). La construcción de identidades docentes como constructo de estructura y dinámicas: argumentación y virtualidades teóricas y prácticas. Profesorado, revista de currículum y formación del profesorado, 11(3). https://tuit.cat/kxmnT

Lorenzo, J. A. (2010). La formación práctica del magisterio: perspectivas. CEE Participación Educativa, 15, 26-39. https://cutt.ly/CkCZQvc

Madalena, J. I., Pinheiro, M. F., Rodríguez, L. A. y Souto, X. M. (2020). La valoración del Practicum en la formación inicial: un estudio de casos internacional en el área de Geografía e Historia. Revista Interuniversitaria de Formación del Profesorado, 95(34.3), 97-118. https://doi.org/10.47553/rifop.v34i3.81004

Masgrau-Juanola, M., López-Ros, V., Serra-Bonet, J. M. y Falgàs-Isern, M. (2017). The practicum model in Teacher Education studies of the University of Girona: connecting theory and practice. En J. Mena, A. García-Valcárcel, F. J. GarcíaPeñalvo y M. Martín del Pozo (eds.), Search and research: teacher education for contemporary contexts (pp. 536-543). Ediciones de la Universidad de Salamanca. https://cutt.ly/WkXY98S 
McIntyre, J. y Hobson, A.J. (2016). Supporting beginner teacher identity development: external mentors and the third space. Research Papers in Education, 31(2), 133158. https://doi.org/10.1080/02671522.2015.1015438

Munday, J., Rowley, J. y Polly, P. (2017). The use of visual images in building professional self identities. International Journal of ePortfolio, 7(1), 53-65. http://www.theijep.com/pdf/IJEP238.pdf

Myers, Ch. G. (2018). Coactive vicarious learning: toward a relational theory of vicarious learning in organizations. Academy of Management Review, 43(4), 610634. https://doi.org/10.5465/amr.2016.0202

Myers, Ch. G. (2020). Performance benefits of reciprocal vicarious learning in teams. Academy of Management Journal, 0(0). https://doi.org/10.5465/amj.2018.0875

Poveda, B., Barceló, M. L., Rodríguez Gómez, I. y López-Gómez, E. (2021). Percepciones y creencias del estudiantado universitario sobre el aprendizaje en la universidad y en el prácticum: un estudio cualitativo. Revista Complutense De Educación, 32(1), 41-53. https://doi.org/10.5209/rced.67953

Saldana, J. (2016). The coding manual for qualitative researchers (3rd ed.). SAGE.

Silverman, D. (2014). Interpreting qualitative data (4th edition). SAGE.

Rodríguez Illera, J. L. y Anaccontini, G. (coords.) (2019). Metodologías educativas en educación. Edicions de la Universitat de Barcelona. https://cutt.ly/GkCZbih

Steenekamp, K., Van der Merwe, M. y Mehmedova, A.S. (2018). Enabling the development of student teacher professional identity through vicarious learning during an educational excursion. South African Journal of Education, 38(1). https://doi.org/10.15700/saje.v38n1a1407 
